\title{
Il y a parallèle et parallèle : la comparaison du mur romain et du mur macédonien chez Polybe $(18,18$, 2-18) et Tite-Live (33, 5, 5-12)
}

\section{Benoît Sans}

\section{CpenEdition}

Journals

Édition électronique

URL : https://journals.openedition.org/rhetorique/1115

DOI : 10.4000/rhetorique.1115

ISSN : 2270-6909

Éditeur

UGA Éditions/Université Grenoble Alpes

Édition imprimée

ISBN : 978-2-37747-296-3

Référence électronique

Benoît Sans, « II y a parallèle et parallèle : la comparaison du mur romain et du mur macédonien chez Polybe (18, 18, 2-18) et Tite-Live (33, 5, 5-12) », Exercices de rhétorique [En ligne], 16 | 2021, mis en ligne le 17 mai 2021, consulté le 19 mai 2021. URL : http://journals.openedition.org/rhetorique/1115 ; DOI : https://doi.org/10.4000/rhetorique.1115

Ce document a été généré automatiquement le 19 mai 2021.

\section{c) (ㅇ)(2)}

Les contenus de la revue Exercices de rhétorique sont mis à disposition selon les termes de la Licence Creative Commons Attribution - Pas d'Utilisation Commerciale - Partage dans les Mêmes Conditions 4.0 International. 


\title{
Il y a parallèle et parallèle : la comparaison du mur romain et du mur macédonien chez Polybe (18, $18,2-18)$ et Tite-Live $(33,5,5-12)$
}

\author{
Benoît Sans
}

\section{Introduction : historiographie et instruction rhétorique}

1 Dans le domaine des études classiques, les sources rhétoriques, en particulier celles qui touchent à son enseignement dans l'Antiquité, comme les manuels de progymnasmata, font désormais l'objet d'un regain d'intérêt certain, grâce à des éditions récentes, des études nouvelles ${ }^{1}$, mais aussi grâce à leur étonnante actualité. Dans des sociétés où l'importance de la capacité à communiquer, convaincre, critiquer ou "performer » à l'oral se fait tous les jours ressentir, l'idée que la pratique des exercices anciens, en adaptant le matériel fourni par les Anciens, pourrait encore aider à développer des compétences techniques et critiques pour la production ou l'analyse de discours contemporains, a même commencé à faire son chemin dans de nombreuses universités ${ }^{2}$, ainsi que dans la présente revue ${ }^{3}$. Ce numéro consacré à l'exercice du parallèle confirme la tendance. Mais redécouvrir la formation des Anciens, c'est aussi envisager ses sources et l'influence que celle-ci a pu exercer dès l'Antiquité sur des générations d'auteurs et leurs œuvres. Les sources sur l'enseignement de la rhétorique nous permettent ainsi de relire des pans entiers de la littérature antique ou, comme dans cet article, de reprendre de précédentes recherches ${ }^{4}$ avec un regard et des outils conceptuels nouveaux.

2 Si cette influence de la formation à la rhétorique a déjà été bien mise en évidence dans certains genres, avec lesquels la rhétorique entretient des liens étroits, comme la prose oratoire ou épistolaire, les romans grecs et latins ou encore certaines formes de poésie, 
on s'est encore peu interrogé sur ses liens avec l'historiographie. On voit pourtant dans les manuels que l'historiographie était abondamment exploitée par cette instruction ${ }^{5}$, les rhéteurs y puisant des exemples, pour illustrer certains procédés mis en œuvre dans les exercices, ou même des sujets et des idées d'énoncés. Il est donc légitime de s'interroger en retour sur les effets que cette formation a pu avoir sur l'écriture de l'histoire ${ }^{6}$. Mais les exemples tirés de l'historiographie soulèvent aussi la question de l'inspiration des rhéteurs pour théoriser des procédés et élaborer les exercices susceptibles de les faire pratiquer. C'est sous cet angle particulier que nous aimerions aborder à nouveaux frais les récits de Polybe et Tite-Live sur la bataille de Cynoscéphales, qui opposa en 198 avant J.-C. l'armée romaine commandée par Titus Quinctius Flamininus à celle de Philippe $\mathrm{V}$ de Macédoine. Comme nous le verrons, ces récits sont en liens étroits avec l'exercice du parallèle. Nous analyserons en particulier les comparaisons du mur macédonien et du mur romain qui figurent au début des deux extraits, avant d'aborder la relation que celles-ci entretiennent avec les récits dans lesquels elles s'insèrent.

3 En tant qu'exercice indépendant, le parallèle ( $\sigma u ́ \gamma \kappa p \iota \sigma \iota \varsigma)$, qui consistait en une sorte de double éloge ou blâme, semble avoir été intégré de façon relativement récente dans le cycle des progymnasmata ${ }^{7}$. Une explication pourrait être que cet exercice s'appuie sur les lieux déjà exercés dans le cadre de l'éloge et du blâme et qu'une forme de parallèle est déjà présente dans le plan de l'éloge et du lieu commun. On trouve des traces de ces discussions dans les manuels conservés, qui s'efforcent alors de distinguer le parallèle de l'éloge et des autres exercices ${ }^{8}$. Il n'est toutefois pas difficile de trouver des exemples antérieurs de cette pratique, qui était notamment en vogue chez les philosophes d'époque hellénistique ${ }^{9}$. L'historien grec Polybe avait probablement baigné dans cette ambiance et, en tant que membre de l'élite de son temps, avait certainement reçu une formation à la rhétorique, même s'il est difficile d'en connaître le contenu. Quoi qu'il en soit, comme l'avait déjà compris Paul Pédech ${ }^{10}$, mais comme l'ont surtout montré des études plus récentes ${ }^{11}$, rhétorique et argumentation sont très présentes dans les Histoires, et cela même si l'historien grec critique ses prédécesseurs pour leur usage abusif des techniques enseignées par les rhéteurs. Pédech montre aussi que la comparaison elle-même est au cœur du projet de l'historien grec ${ }^{12}$ qui, à travers une histoire universelle abordant le destin de différents peuples, cherche à comprendre comment Rome a pu accéder au premier rang et imposer sa domination aux autres (Pol. 1, 1-2). La comparaison est aussi inscrite dans sa "méthode historique ${ }^{13}$ »: il a fréquemment recours aux analogies avec d'autres domaines ou exemples historiques ${ }^{14}$; il compare les cités et leur situation géographique, les organisations militaires, les systèmes politiques et, bien avant Plutarque, les qualités et les défauts des protagonistes de son œuvre $^{15}$. Si Pédech veut voir dans cette abondance de comparaisons le signe d'une méthode "véritablement historique ${ }^{16}$ ", éloignée des amplifications de la rhétorique, il est forcé de constater que bon nombre de ces comparaisons semblent en lien étroit avec l'antique discipline et plus particulièrement, avec le genre épidictique ${ }^{17}$. C'est que l'éloge et le blâme font aussi partie de la tâche de l'historien, telle que la présente Polybe ${ }^{18}$, et faisaient vraisemblablement déjà l'objet d'exercices ${ }^{19}$. On retrouve là les principaux éléments de l'exercice du parallèle tel qu'il apparaît dans les manuels de progymnasmata - car toute comparaison n'est pas parallèle rhétorique. Il n'y avait plus pour Polybe qu'un petit pas à faire et qui avait déjà pu être franchi. 
Or tous ces éléments se retrouvent concentrés de façon spectaculaire et à petite échelle dans la manière dont l'historien grec traite de la bataille de Cynoscéphales (18, 18-32). La narration est ici littéralement encadrée par la comparaison: il y a d'une part le parallèle du mur macédonien et du mur romain au début du récit, sur lequel nous nous pencherons un peu plus loin, mais c'est aussi le moment que choisit Polybe pour confronter la tactique de la phalange gréco-macédonienne et celle de l'armée romaine, dans un long commentaire placé juste après le récit de la bataille. Dans cette dernière partie, Polybe $(18,28-32)$ livre une argumentation en règle en faveur de la supériorité tactique des Romains, dont la partie centrale consiste en une double description des dispositifs des deux armées. La démarche est à la fois rigoureuse et habile pour convaincre et ménager un public grec: après avoir écarté l'idée que, comme dans la guerre précédente, ce sont les qualités des chefs qui ont été déterminantes, Polybe confronte les tactiques et montre dans un premier temps qu'aucune armée ne peut résister au choc de la phalange ; mais il précise que cela n'est valable que lorsque les conditions sont réunies pour que la phalange puisse déployer toute sa puissance $(18,30,11)$. Il montre ensuite que de nombreux éléments - un terrain accidenté ou comportant des obstacles, une tactique fuyante de l'ennemi... - peuvent entraver la phalange et la mettre en échec. Or un de ces critères est toujours réalisé dans le dispositif romain : celui-ci inclut des réserves et procède par assauts successifs, tandis que la phalange ne peut effectuer qu'une seule charge ; une fois engagée dans un corps à corps, cette dernière peut facilement être contournée. Et l'historien grec de conclure la comparaison sur la supériorité de la tactique romaine.

5 Comme nous l'avons annoncé, un autre élément rend la bataille de Cynoscéphales particulièrement pertinente pour l'étude du parallèle. Elle fait partie des épisodes pour lesquels il est possible de lire également le récit de Tite-Live, et même de ceux où l'historien latin semble s'être servi des Histoires de manière assez étroite pour son propre récit ${ }^{20}$. Il est donc possible de comparer les deux auteurs dans leur façon de rapporter le même événement. Un indice qui plaide fortement en faveur de l'utilisation de Polybe comme source est justement que Tite-Live choisit de récupérer le parallèle qui figure au début du récit de son modèle grec. Tite-Live, qui n'est pas si éloigné de Quintilien et des progymnasmata ${ }^{21}$, avait, plus probablement encore que Polybe, été formé à la rhétorique et a parfois été soupçonné d'avoir été rhéteur lui-même ${ }^{22}$; et le parallèle ne lui est pas non plus étranger ${ }^{23}$. L'épisode de Cynoscéphales permet ainsi de confronter les deux auteurs dans un même exercice et, plus largement, dans leur pratique de l'antique discipline.

\section{D'un parallèle à l'autre : les comparaisons du mur macédonien et du mur romain}

$6 \quad$ Les deux textes se recoupent au moment où Flamininus décide de partir à la poursuite de Philippe et ordonne à ses troupes d'emporter des pieux pour les fortifications du camp. C'est cette instruction qui engendre chez Polybe $(18,18,1)$ une discussion comparative sur les pratiques des Grecs et des Romains en la matière $(18,2-18)^{24}$ :

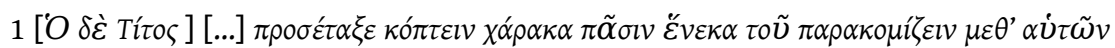

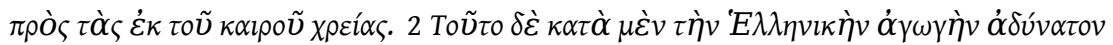

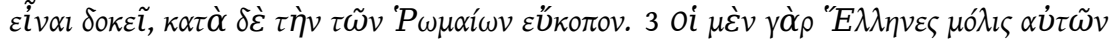

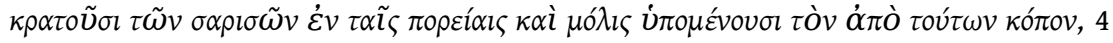




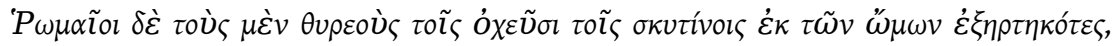

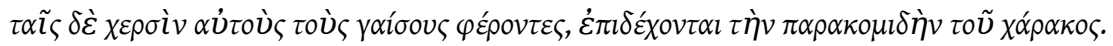

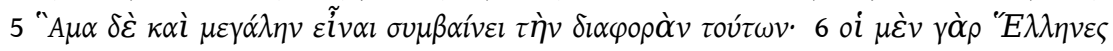

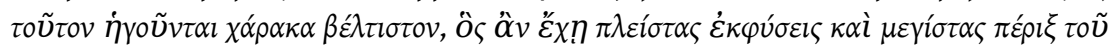

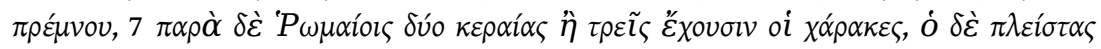

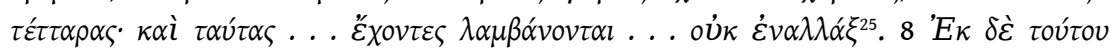

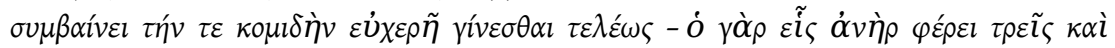

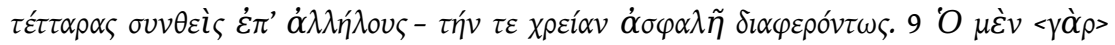

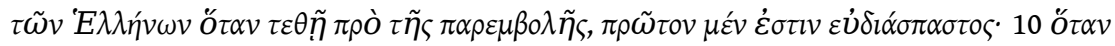

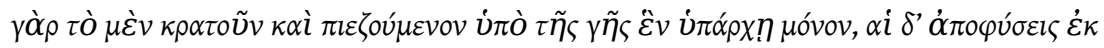

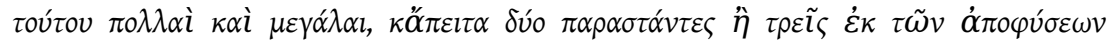

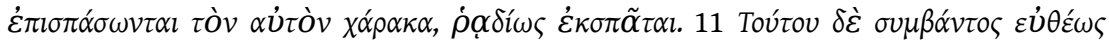

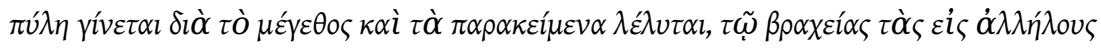

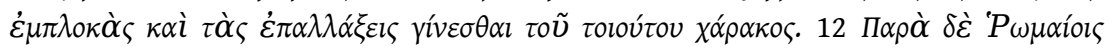

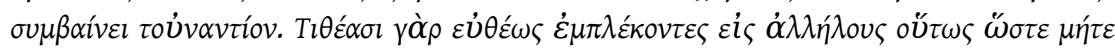

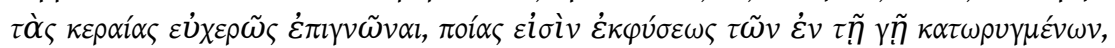

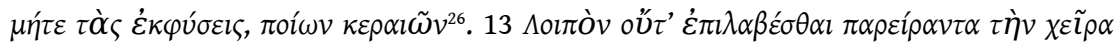

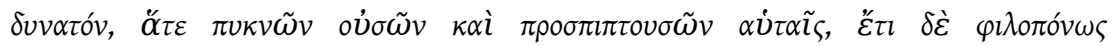

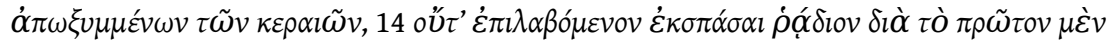

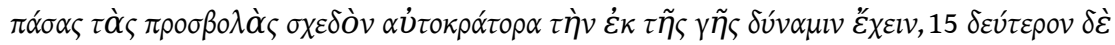

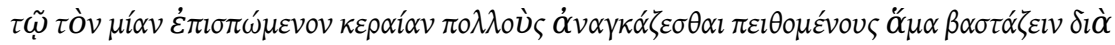

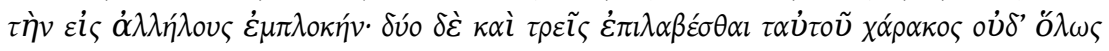

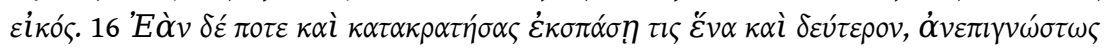

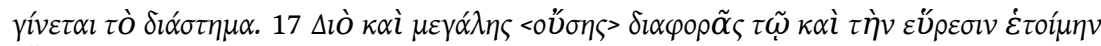

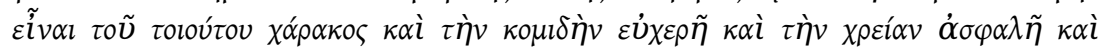

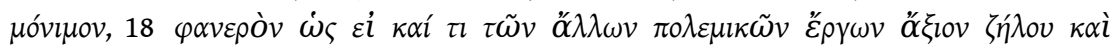

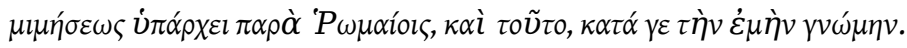

1 [...] Il ordonna donc à tous ses hommes de tailler des pieux, qu'ils transporteraient avec eux, pour le cas où l'on en aurait besoin. 2 Dans une armée équipée à la grecque, un tel ordre eût paru impossible à exécuter, mais il ne présentait aucune difficulté pour les troupes romaines. 3 En effet, les Grecs ont déjà assez de peine à porter leurs sarisses au cours des marches et à endurer la fatigue que leur impose une telle charge. 4 Mais les Romains, portant leurs boucliers suspendus à leurs épaules par des courroies et ne tenant à la main que leurs javelots, peuvent porter en outre des pieux. 5 Qui plus est, les pieux qu'ils taillent sont d'un type particulier. 6 Alors que les Grecs considèrent que les meilleurs sont ceux qui portent, autour du tronc principal, les branches les plus nombreuses et les plus longues, 7 les Romains les choisissent avec deux, trois ou, tout au plus, quatre branches... [qui ne partent pas dans toutes les directions, mais s'ouvrent sur un seul plan], 8 ce qui les rend très faciles à transporter - un seul pouvant en prendre avec lui deux ou trois, liés en faisceau; ce qui fait aussi que ces pieux assurent une protection incomparable. 9 Ceux des Grecs, quand ils sont plantés en avant d'un camp, sont d'abord très faciles à arracher. 10 Comme la seule tige maitresse se trouve tenue par la terre, dans laquelle elle s'enfonce, et que les branches qui s'en détachent sont nombreuses et longues, il suffit que deux ou trois hommes en saisissent quelques-unes et tirent sur le pieu, pour que celui-ci soit arraché sans difficulté. 11 Cela fait, c'est aussitôt une véritable porte qui se trouve ouverte, étant donné la place qu'occupe chaque pieu. En outre les pieux voisins ne tiennent plus, parce que, dans une palissade ainsi conçue, ils ne sont rattachés les uns aux autres que par les quelques rares branches qu'on a pu entrelacer. 12 Chez les Romains, il en va tout à l'inverse. Ils plantent, en effet, leurs pieux de façon à former un tel enchevêtrement qu'il est difficile de distinguer quelles sont les branches qui se rattachent à telle souche ou quelle est la souche qui porte telle branche. 13 Ainsi, comme leurs branches forment, en s'entrecroisant, un réseau serré et que leurs extrémités ont été soigneusement taillées en pointe, il est impossible d'y glisser la main pour saisir un des pieux. $14 \mathrm{Et}$, 
à supposer qu'on y parvienne, il est bien difficile de l'arracher, d'abord parce que, de quelque côté qu'on s'attaque à lui, il est si bien planté en terre qu'il résiste par lui seul à tous les efforts, 15 ensuite parce que celui qui tire sur une seule de ses branches doit en même temps venir à bout de plusieurs autres pieux, qui tiennent au premier, par l'entrelacement de leurs ramures. 16 Et il n'arrive pas, normalement, que deux ou trois hommes s'attaquent au même pieu. D'autre part, si un assaillant vient à bout d'un ou deux pieux, la brèche ainsi ouverte est à peine perceptible. 17 Telle étant la supériorité de ce type de pieu, qui est facile à transporter et qui fait des palissades solides et sûres, 18 nous avons là, parmi toutes les pratiques militaires des Romains, un procédé qui mérite, plus que tout autre, d'être étudié et imité. C'est du moins mon opinion.

7 Le parallèle ainsi proposé par Polybe présente de nombreux points communs avec l'exercice du même nom, et à plusieurs niveaux. Pour ce qui concerne le contenu et l'argumentation, si les objets comparés peuvent paraitre inhabituels au regard des thèmes attestés, et même si Polybe désigne clairement un vainqueur, les deux murs apparaissent comparables, comme les deux armées en présence, et cela d'autant plus qu'ils relèvent d'une même catégorie. On observe divers moyens d'amplification et une organisation à la fois chronologique et logique des différents points: le premier concerne l'origine des pieux et la facilité de leur transport ; cette dernière propriété est mise en lien avec les qualités intrinsèques des pieux; le reste du propos porte sur l'efficacité des pieux et leurs conséquences une fois le mur constitué. Moyennant un effort d'analogie ${ }^{27}$, il est possible d'y reconnaître les lieux de l'éloge, qui s'appliquent dans le parallèle. Mais la proximité est plus grande encore au niveau de la structure : en comparant les deux murs point par point, Polybe adopte la disposition qui sera recommandée par les manuels de progymnasmata. On retrouve en outre pour chaque critère une structure récurrente : une affirmation anticipative est chaque fois justifiée ( $\gamma \alpha_{\rho} \rho$, « en effet») par la comparaison proprement dite ; chaque argument est ainsi appuyé par un ou plusieurs sous-arguments généralement soulignés par un

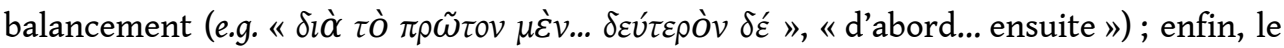
point s'achève par un résultat concret qui reformule l'affirmation initiale ${ }^{28}$ : le soldat romain peut ainsi emporter plus de pieux que le soldat gréco-macédonien; il suffit de peu d'hommes pour démanteler le mur gréco-macédonien et créer presque instantanément une ouverture béante, tandis que le mur romain présente un tel entrelacement qu'il est difficile de s'emparer des pieux et de distinguer les troncs, qu'il faut de nombreux hommes pour en venir à bout et que la brèche est à peine perceptible. En outre, bien qu'il soit inséré de façon assez naturelle dans le récit, le parallèle est clôturé par une conclusion explicite $(18,17:$ : $i$, « Telle étant ») reprenant les différents points et invitant à l'imitation du modèle romain. Enfin, sur le plan linguistique et stylistique, le texte est très soigné : les nombreux balancements, les degrés de comparaison, les ordres de grandeur, les antithèses et les diverses répétitions structurent et renforcent les oppositions; d'autres jeux de figures (chiasmes, hyperbates) accompagnent le propos (notamment pour évoquer l'enchevêtrement des pieux). Ce sont aussi des traits attendus de ce genre de discussion, que l'on retrouve dans les modèles fournis par les manuels.

8 Au même endroit de son récit, Tite-Live $(33,5,5-12)$ récupère et condense à la fois le contenu du texte grec de la façon suivante ${ }^{29}$ :

5 Vallo et Macedones et Graeci usi sunt, sed usum nec ad commoditatem ferendi nec ad ipsius munitionis firmamentum aptauerunt; 6 nam et maiores et magis ramosas arbores caedebant quam quas ferre cum armis miles posset, et cum castra his ante obiectis 
saepsissent, facilis molitio eorum ualli erat. 7 Nam et quia rari stipites magnarum arborum eminebant multique et ualidi rami praebebant, quod recte manu caperetur, 8 duo aut summum tres iuuenes conixi arborem unam euellebant, qua euulsa portae instar extemplo patebat, nec in promptu erat quod obmolirentur. 9 Romanus leues et bifurcos plerosque uel trium aut cum plurimum quattuor ramorum uallos caedit, ut et suspensis ab tergo armis ferat plures simul apte miles; 10 et ita densos obfigunt implicantque ramos ut neque <quis cuiusque palmae stipes neque ${ }^{30}$ quae cuiusque stipitis palma sit, peruideri possit; 11 et adeo acuti alius que per alium immissi rami locum ad inserendam manum non relinquunt, 12 ut neque prehendi quod trahatur neque trahi, cum inter se innexi rami uinculum in uicem praebeant, possit; et si euulsus forte est unus, nec loci multum aperit et alium reponere perfacile est.

5 Les Macédoniens et les Grecs employaient ces pieux, mais ils ne les avaient pas adaptés pour les transporter aisément et pour renforcer leur efficacité défensive. 6 En effet, ils les coupaient trop grands et leur laissaient trop de branches pour qu'un soldat arrivât à les porter avec ses armes et, quand ils avaient entouré le camp en les plaçant en avant, la démolition de la palissade qu'ils constituaient était facile. 7 En effet, comme les troncs de ces grands arbres faisaient saillie de loin en loin et que des branches nombreuses et solides offraient de bonnes prises, 8 deux jeunes soldats, ou trois tout au plus, en unissant leurs forces, parvenaient à arracher un arbre, qui laissait aussitôt, une fois arraché, un passage grand comme une porte, sans qu'il y eût à côté quoi que ce soit pour réparer. 9 Les Romains, eux, coupent des pieux légers, à deux branches, le plus souvent, voire à trois ou quatre au plus, de façon que le soldat, en se suspendant les armes sur le dos, en porte aisément plusieurs en même temps. 10 Ils les plantent si serrés et si imbriqués que personne ne peut repérer ni quel tronc correspond à chaque branche ni quelle branche correspond à chaque tronc. 11 Ces branches pointues, entrelacées l'une dans l'autre, permettent si peu de passer la main que l'on ne parvient pas à les saisir pour les tirer, ni à les tirer, puisqu'elles sont enchevêtrées et liées l'une à l'autre. Si par hasard l'un des pieux est arraché, il n'ouvre pas une grande brèche et il est aisé de le remplacer.

On remarque immédiatement quelques différences avec le modèle grec: Tite-Live ne propose pas de conclusion explicite et redistribue le contenu en traitant en premier lieu tout ce qui concerne les Grecs, immédiatement associés aux Macédoniens, et en second lieu, ce qui concerne les Romains. Pour le mur grec, l'historien latin $(5,5)$ corrige d'emblée l'affirmation initiale et refuse au rempart gréco-macédonien, les qualités attendues : il est inadapté pour le transport et ne fournit pas une protection efficace. Tite-Live justifie ensuite («nam et», « en effet») la première partie de la conclusion qu'il défend, puis un peu plus loin $(5,7)$ la seconde («facilis molitio », " une démolition facile») avec ses conséquences et la même structure ("Nam et », "en effet »). Certains termes et structures rappellent le texte grec (deux raisons ou causes admises sont introduites par quia, parce que, et reliées par et pour former un parallélisme ; même image que chez Polybe pour évoquer la brèche, "portae instar ", "comme une porte»). Toutefois, le texte latin accentue par les mots et le style (polyptote et anadiplose dans « euellebant quae euulsa parte ", " parvenaient à arracher... une fois arraché ", adverbe "extemplo", " aussitôt ») l'aisance avec laquelle un faible nombre d'hommes peut démonter une partie de la palissade puis accentue l'immédiateté du résultat. Il ajoute aussi un élément supplémentaire : il n'y a alors aucun moyen à disposition pour colmater la brèche ( nec in promptu erat quod obmolirentur ", "sans qu'il y eût à côté quoi que ce soit pour réparer »). Pour le mur romain $(5,9)$, dont les différentes qualités sont reliées par et, Tite-Live accumule d'abord les adjectifs en gradation pour conclure à la facilité du transport. Ensuite, le réseau de branches enchevêtrées et le résultat de cette disposition rappellent la 
formulation du texte grec: chiasme, polyptote et effets sonores évoquent l'impossibilité de discerner les branches et les troncs. L'historien latin analyse ensuite la résistance offerte par le dispositif complet avec une structure syntaxique, rythmique et logique tout à fait parallèle ("et ita densos... ut neque... neque ", "si serrés et si imbriqués que personne ne peut... ni »; «et adeo acuti... ut neque... neque », «si pointues et entrelacées... que... ni »), divers effets stylistiques renforcent la persuasion: polyptotes ("alius per alium », "l'une dans l'autre», "trahatur... trahi », «à les saisir pour les tirer, ni à les tirer »), paronomase (« immissi rami... innexi rami », « ces branches entrelacées... enchevêtrées »), homéotéleutes (retour de la finale -i), allitération (" uinculum in uicem », « et liées l'une à l'autre »). Enfin, l'éventualité d'une brèche dans le mur est rendue encore plus improbable par l'adverbe forte, la conséquence est négligeable ("nec loci multum aperit», «il n'ouvre pas une grande brèche»), et on retrouve le pendant de l'élément supplémentaire introduit plus tôt : contrairement au dispositif gréco-macédonien, il est très facile de combler l'intervalle créé par le pieu manquant dans le mur romain (" et alium reponere perfacile est », " et il est aisé de le remplacer »).

Si la conclusion est ici laissée au lecteur - un procédé bien connu pour renforcer sa conviction $^{31}-$, celle-ci est sans appel. Le texte latin creuse davantage l'écart par les amplifications, par des éléments supplémentaires et par la structure même du propos. Bien qu'une telle disposition semble admise par Théon (Prog. 10, 82 Patillon), elle sera explicitement rejetée par les manuels ultérieurs. Aphthonios (Prog. 10, 3, 141 Patillon)

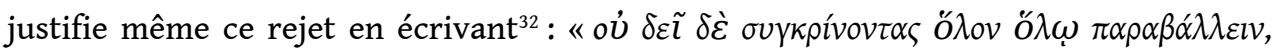

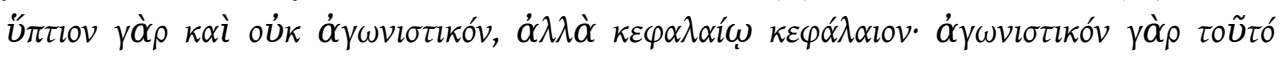
$\gamma \varepsilon »$ ( « il ne faut pas comparer en rapprochant un ensemble et un autre, car cela est plat et non agonistique, mais plutôt un point et un autre, car c'est cela qui est agonistique »). Selon le rhéteur, la disposition adoptée par Tite-Live viderait donc l'exercice de son intérêt et, sans doute, de son attrait pour le lecteur. Elle pourrait cependant correspondre à une formation aux progymnasmata relativement « jeune » et qui aurait récemment intégré le parallèle. Mais le passage soulève un problème plus large. En effet, si la présence de ce passage dans la version livienne de l'épisode peut être considérée comme un indice fort du fait que Tite-Live s'est servi de Polybe comme source, l'extrait apparaît comme une digression technique, bien conforme à la manière et aux préoccupations de Polybe, mais n'a pas d'impact direct sur la causalité des événements et sur l'issue de la bataille. On peut dès lors s'interroger sur l'intérêt pour Tite-Live de reprendre, contrairement à ses habitudes, un tel extrait dans son propre récit; le même Tite-Live ne récupère rien ou presque ${ }^{33}$ de la discussion qui suit immédiatement le récit de l'historien grec et qui apparaît bien plus pertinente pour comprendre l'événement. Les différences observées permettent d'exclure un recopiage servile ou maladroit de la part de l'historien latin et poussent à chercher d'autres raisons dans le reste de l'épisode.

\section{Cynoscéphales ou le récit de bataille comme parallèle}

10 La bataille est par excellence le moment qui permet de soupeser les forces et les faiblesses des armées en présence ${ }^{34}$, et Polybe a clairement placé son récit de l'épisode de Cynoscéphales sous l'angle de ce genre de questionnement, qui a pu entourer la 
bataille. Si l'on en croit les récits fournis par les deux auteurs, celle-ci n'a rien de la bataille rangée "classique»: les deux adversaires progressent longtemps sans se trouver, rencontrent divers obstacles et cherchent des informations sur la position de l'autre, ce qui provoque diverses escarmouches, avant que l'une de celles-ci n'évolue, de façon inattendue, en un affrontement général. Mais à chaque étape du récit, les deux auteurs laissent ainsi apprécier à tour de rôle les réactions des deux belligérants face à des objectifs ou des difficultés communes.

11 Chez Polybe, au cours des jours qui précèdent la bataille, les deux chefs d'armée réagissent de façon similaire aux mêmes facteurs: les encombrements du terrain, l'apparition d'un épais brouillard, le besoin de ravitaillement. Et si Philippe s'aventure imprudemment dans le brouillard $(20,8)$, il parvient à rattraper la situation. Polybe est le seul à préciser qu'il cherche obstinément un terrain adapté à ses troupes ${ }^{35}(20,2)$. Faisant ainsi preuve d'une égale compétence, aucun des deux commandants ne semble prendre l'avantage. Le résultat des premières escarmouches apparaît à peu près nul : dans un cas, l'affrontement n'a pas lieu; dans l'autre, le détachement romain, mis en difficulté par de vaillants Macédoniens, est sauvé par l'intervention de cavaliers étoliens, dont Polybe souligne les qualités tactiques ${ }^{36}(22,5)$.

Les deux chefs d'armées sont pris au dépourvu par la rencontre inattendue de deux détachements et par l'escalade des hostilités. Philippe est trompé par les rapports encourageants de messagers. Polybe $(22,8)$ transmet ceux-ci en style direct, ce qui crée un effet de point de vue et rend la méprise plus compréhensible. Le roi semble ici particulièrement gêné par le fait que le lieu ne correspond pas à ses attentes ${ }^{37}(22,10)$. Bientôt, il constate également $(24,7)$ qu'une partie de ses troupes accuse un certain

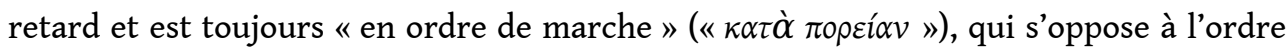
de bataille. Tandis que Flamininus a remotivé ses troupes par une brève harangue et s'apprête à lancer l'assaut (23), le souverain macédonien, occupant une position surélevée, ne se laisse pas abattre et réorganise rapidement une phalange avec les troupes qu'il a sous la main. Là où elle profite d'une position et de conditions avantageuses $(25,2)$, la phalange l'emporte, grâce à sa force et à son équipement selon Polybe, et l'issue semble encore indécise.

13 Ce sont alors les faiblesses de la phalange qui vont précipiter la défaite de Philippe. Flamininus décèle une faille dans le dispositif désordonné de son adversaire, et Polybe (25, 3-5) met bien en évidence les facteurs qui guident la décision du général romain : ce dernier lance l'assaut sur les troupes qui ne sont pas rangées et qui se débandent immédiatement. Polybe (25, 6-7) s'attarde longuement sur cette déroute et la justifie en précisant que les troupes n'avaient pas reçu d'ordre et n'avaient encore pu former la

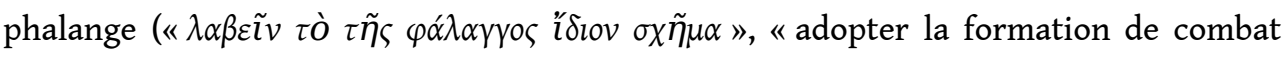
propre à la phalange »). Sur l'autre flanc, la phalange de Philippe tient bon, mais une autre faiblesse est opportunément exploitée par un tribun romain ${ }^{38}$. Polybe affirme

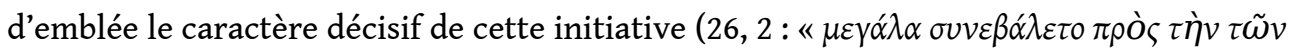
ó $\lambda \omega v \kappa \alpha \tau o ́ \rho \theta \omega \sigma l v »$, «Il contribua ainsi pour une large part au succès final »), puis la justifie en détaillant l'action du tribun: avec un faible nombre d'hommes et une certaine facilité, il exécute une manœuvre de contournement et prend la phalange au piège ; celle-ci est rapidement dispersée et massacrée. Alors que le roi est en train de se replier, Polybe nous livre une dernière fois ses pensées $(26,8)$ : Philippe comprend qu'il a été vaincu, car il a laissé à l'adversaire une position avantageuse qu'il occupait. 
14 Dans la version latine de l'épisode, si les deux protagonistes sont dépeints dans les mêmes activités, leurs réactions apparaissent légèrement différentes. Flamininus ${ }^{39}$ se montre prudent, redoutant les manœuvres de son adversaire et les embuscades ${ }^{40}$, là où Philippe est pressé d'en découdre $(6,3)$ et s'obstine. Les différents obstacles rencontrés donnent ici lieu à de courtes séquences descriptives plus détaillées que dans le texte grec: les troupes de Philippe sont ainsi représentées en train d'errer un temps à l'aveuglette au milieu d'un épais brouillard $(7,1)$. De la même façon, lors des premières rencontres, le choix et le contraste des expressions font que les Romains apparaissent vainqueurs ou peu diminués, tandis que les Macédoniens paraissent plus enfoncés et leurs reculs sont amplifiés ${ }^{41}$.

Tite-Live $(8,1)$ affirme immédiatement, et plus tôt que dans le texte grec, le caractère erroné des messages reçus par Philippe et sanctionne ainsi son erreur d'appréciation. Chaque fois trompé dans son attente, le souverain macédonien apparaît ici davantage troublé et hésitant, et ses réactions appartiennent davantage au registre émotionnel qu'à celui de l'analyse tactique ${ }^{42}$. Il se retrouve bien vite dans des situations qui semblent plus compliquées que dans le texte grec: il est d'abord contraint de se battre pour sauver les troupes qui sont déjà engagées $(7,10)$, puis bientôt, pour sauver même sa propre vie $(8,11$ : "ne ipsi quidem in tuto iam receptus erat ", " même pour lui, il n'y avait plus de retraite sûre »). Il donne l'impression de subir le cours des événements plus que son adversaire. L'historien latin s'étend moins sur les réactions de Flamininus : celui-ci semble simplement mieux s'adapter à la situation avec des troupes qui récupèrent vite. Au cœur de la bataille, la phalange réorganisée par Philippe, n'a ici pour seul avantage ${ }^{43}$ que sa position surélevée (« loci plurimum auxilio ", " en large partie avec l'aide du terrain »), mais ce petit succès est noyé au milieu d'informations négatives et d'un tableau de bataille globalement défavorable pour Philippe ${ }^{44}(9,3-4)$. Lorsque le Romain lance son assaut décisif $(9,6-7)$, la moitié de l'armée macédonienne succombe à la peur des éléphants ("terrore primo bestiarum auersi ", «immédiatement, les Macédoniens tournèrent le dos, mis en fuite par la frayeur, dès qu'ils virent les animaux ») et l'heureuse initiative du tribun romain $(9,8-9)$ ne fait ici qu'accélérer et justifier une défaite déjà annoncée par le narrateur («Et ceteri quidem hos pulsos sequebantur ", " Et tous les suivirent dans leur déroute »).

16 En résumé, à la manière de l'enquêteur judiciaire, Polybe est à la recherche des causes et se sert du parallèle pour identifier avec précision les facteurs véritablement déterminants et en éliminer d'autres ${ }^{45}$. Tout est fait dans le texte grec pour mettre en exergue les données tactiques, qui apparaissent comme les véritables causes de la défaite de Philippe : les autres facteurs, que ce soit les techniques de fortification, les aléas de la progression ou la personnalité des deux chefs d'armées ${ }^{46}$, sont pour ainsi dire neutralisés ${ }^{47}$; les deux forces en présence paraissent ainsi soutenir la comparaison jusqu'à ce que leurs spécificités viennent les départager; ces différences sont ainsi révélées par la confrontation et la mise en parallèle systématique des actions de chacun, qui vient s'associer à la logique du récit. Celui-ci est mis au service des conclusions que l'auteur veut porter dans l'argumentation qui suit, selon un dispositif qui rappelle fortement la narratio et l'argumentatio d'un discours. Le lecteur est ainsi emmené dans une implacable entreprise de persuasion et comprend finalement qu'en raison des spécificités tactiques des deux armées, le combat était joué d'avance. Toutefois, en adoptant une dispositio par critères dans le parallèle initial, en retardant l'apparition des facteurs qui précipitent la défaite de Philippe, et en admettant que la 
phalange est imbattable sous certaines conditions, Polybe rend les deux armées comparables, maintient une certaine tension narrative et ménage peut-être la sensibilité d'un public grec, qui n'a pas encore tout à fait digéré qu'une tactique pourtant éculée ait pu être mise en échec par les Romains. Partant, l'historien grec invite à tirer les leçons du passé et à s'en inspirer pour l'avenir ${ }^{48}$.

L'historien latin simplifie le raisonnement porté par le récit en construisant à chaque étape un contraste constant entre les deux armées : les Romains, efficaces sans être particulièrement brillants, semblent ainsi l'emporter parce qu'ils affrontent de piètres adversaires, qui accumulent les désavantages, sont soumis à leurs émotions et dirigés par un chef plus fougueux que compétent. Dans les deux cas donc, les parallèles sur les fortifications des deux camps s'insèrent bien dans la logique propre voulue par les deux auteurs et en révèlent à l'avance l'orientation. Le commentaire d'Aphthonios sur l'usage du parallèle s'applique bien à la version livienne de l'épisode : au sein d'une œuvre dont la dimension idéologique est bien connue, il ne peut vraiment y avoir de comparaison entre Rome et la Macédoine de Philippe ; autrement et familièrement dit, il n'y a vraiment pas «photo » ou de «match» entre les deux armées. Si dès le départ, l'issue ne fait pas de doute, le récit peut prendre une coloration plus épidictique, qui est aussi plus implicite : il n'est plus une enquête, mais le rappel de hauts faits, émaillés d'effets spectaculaires, rappelant la technique de l'ekphrasis ${ }^{49}$, comme la végétation encombrante qui gêne la progression des troupes $(6,7)$, l'errance des Macédoniens à travers le brouillard $(7,2)$, le massacre des soldats de la phalange $(9,9-11)$, les cadavres et les enseignes romaines à travers les yeux de Philippe $(10,2)$; autant d'éléments que l'historien latin ajoute à son modèle.

Pour exprimer autrement la proximité et la différence entre les deux versions, on peut dire que les deux auteurs donnent une dimension rhétorique à leurs textes et ont chacun à leur manière recours à la technique du parallèle, à l'éloge et au blâme. Cependant, cette rhétorique n'a pas le même objet: tandis que Polybe se concentre en définitive sur un seul critère, Tite-Live continue d'opposer deux groupes et conserve ainsi aux caractères et personnalités en présence un rôle prépondérant.

\section{Conclusion : usages et fonctions du parallèle}

19 S'il n'est pas possible de dire à quel moment les rhéteurs ont thématisé la pratique du parallèle dans un exercice et progressivement intégré celle-ci au sein d'un enseignement à la fois rhétorique et littéraire, ni si les deux auteurs dont il est ici question ont pu être influencés par un tel enseignement, les parallèles étudiés, qui apparaissent déjà très aboutis, sont certainement à verser dans cette histoire. Ils permettent en tout cas d'apprécier les effets et spécificités d'une pratique appliquée à un domaine précis, l'écriture de l'histoire, et peut-être l'intérêt qu'il pourrait y avoir à la réinvestir aujourd'hui.

Le lien entre les parallèles liminaires et les deux récits, mais aussi la raison pour laquelle Tite-Live a conservé, tout en l'adaptant, cette partie de son modèle ne sont pas à chercher au niveau de la causalité historique, mais plutôt à un niveau symbolique, cognitif et argumentatif: les deux parallèles donnent le ton et lancent le récit en invitant le lecteur à adopter une certaine tournure d'esprit qui est ensuite entretenue tout au long du récit. S'il peut paraitre naturel d'associer le parallèle au récit de bataille, il faut constater que les deux auteurs en font un usage différent, au service de 
conclusions ou d'orientations propres, qui témoignent de la diversité et de la richesse de l'exercice.

Dans les deux cas, le parallèle appliqué à l'histoire révèle à la fois une fonction critique et une fonction argumentative et persuasive, qui semblent inextricablement imbriquées: d'un côté, il est une procédure familière et rationnelle qui permet de mieux cerner et organiser une réalité toujours complexe en la rendant plus accessible et plus apte à livrer des enseignements. Dans le même temps, il est un puissant moyen de façonner et de porter une certaine interprétation du réel. Et cette ambivalence n'est autre que celle de la rhétorique elle-même.

\section{NOTES}

1. En ce qui concerne les progymnasmata, voir la récente enquête bibliographique menée par P. Chiron, « Les progymnasmata de l'Antiquité gréco-latine », Lustrum 59, 2017, p. 7-129.

2. Voir plusieurs rapports d'expériences pédagogiques dans P. Chiron, B. Sans, Les progymnasmata en pratique de l'Antiquité à nos jours, Paris, Éditions Rue d'Ulm, 2020.

3. Cf. V. Ferry, B. Sans, Exercices de rhétorique, 5|2015 (numéro intitulé "Rhétorique et citoyenneté »).

4. B. Sans, Narratio probabilis : lectures rhétoriques comparées de Polybe et Tite-Live, Louvain, Peeters, 2020.

5. C. A. Gibson, « Learning Greek History in the Ancient Classroom : the Evidence of the Treatises on Progymnasmata », Classical Philology, 99, 2, 2004, p. 103-129.

6. Voir cependant P. Alvarez, The Impact of Rhetoric and Education on the "Res Gestae" of Amminaus Marcellinus, Edmonton, University of Alberta, 2006 ; M. Bellissime, « Fiction et rhétorique dans les prosopopées de l'"Histoire romaine" : les marges de la liberté de l'historien ", dans V. Fromentin et al., Cassius Dion : nouvelles lectures, Bordeaux, Ausonius, 2016, p. 363-381; dans le même volume :

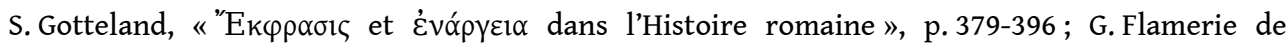
Lachapelle, "Les récits de bataille dans l'œuvre de Florus : enjeux narratifs et idéologiques ", Dialogues d'histoire ancienne, 36, 1, 2010, p. 137-152 ; M. W. Martin, « Progymnastic Topic Lists : a Compositional Template for Luke and Other "Bioi" ?», New Testament Studies, 54, 1, 2008, p. 18-41; B. Sans, «Evadent, inquit: à propos du discours d'Hannibal aux Tarentins (Tite-Live, 25, 11, 16-17) », Latomus, 78, 2019, p. 918-927.

7. M. Patillon, Ælius Théon. Progymnasmata. Texte établi et traduit par M. P., avec l'assistance, pour l'arménien, de G. Bolognesi, Paris, CUF, 1997 [2002], p. lxxxi.

8. Quintilien, Institution oratoire, 2, 4, 21; Ælius Théon, Progymnasmata, 10, 78-82 Patillon; Aphthonios, Progymnasmata, 10, 140-143 Patillon; Pseudo-Hermogène, Progymnasmata, 10, 198-199 Patillon ; Nicolaos, Progymnasmata, 9, 59-63 Felten.

9. G. Reichel, Quaestiones Progymnasmaticae, Leipzig, 1909, p. 96.

10. P. Pédech, La méthode historique de Polybe, Paris, Les Belles Lettres, 1964, p. 43-52.

11. Voir les articles consacrés à Polybe dans le volume édité par H. Verdin, H. Scheppens, E. De Keyser, Purposes of History. Studies in Greek Historiography from the $4^{\text {th }}$ to the $2^{\text {nd }}$ Centuries B. C., Louvain, 1990. Voir également, B. Sans, «Polybe, Tite-Live et la bataille de l'Èbre (Polybe, 11, 31-33 ; Tite-Live, 28, 31, 5 - 34,12) », Revue de Philologie, 2017, 1, p. 165-190 ; B. Sans, « De Zama à 
Cynoscéphales : étude comparée des stratégies rhétoriques de Tite-Live et Polybe ", Livius Noster. Second International Conference on Livy (Padoue, 6-10 Novembre 2017), Padoue, 2020 (à paraître).

12. P. Pédech, op. cit., p. 408.

13. P. Pédech, op. cit., p. 408-431.

14. B. Sans, «L'histoire par l'exemple et l'exemple de l'histoire : les exemples historiques chez Polybe », dans V. Ferry, B. Sans, A. Toma, «Études sur l'exemple », DICE, 8/2, éditions du Musée de la Littérature Roumaine, Bucarest, 2011, p. 138-159.

15. E. Foulon, "Philopoemen, Hannibal, Scipion : trois vies parallèles chez Polybe », Revue des études grecques, 106, 1993, p. 333-379.

16. P. Pédech, op. cit., p. 415.

17. P. Pédech, op. cit., p. 415-416, p. 419, p. 426.

18. Polybe, 1,$14 ; 3,4,7-8 ; 10,21$.

19. Polybe $(12,26$ b , 5) mentionne notamment le blâme de Pénélope ou l'éloge de Thersite parmi les thèmes pratiqués à l'école.

20. Pour une vue d'ensemble des passages parallèles entre Polybe et Tite-Live, voir notamment H. Tränkle, Livius und Polybios, Bâle-Stuttgart, Schwabe \& Co., 1977.

21. Du moins si l'on accepte l'hypothèse de M. Patillon (op. cit., p. vii-xvi), qui s'appuie sur la proximité des programmes proposés par Théon et par le rhéteur latin.

22. Cf. M.-P. Arnaud-Lindet, Histoire et politique à Rome. Les historiens romains (III siècle av. J.-C. - Ve siècle ap. J.-C.), Paris, Bréal, 2001, p. 195 ; E. Cizek, Histoire et historiens à Rome dans l'Antiquité, Lyon, Presses universitaires, 1995, p. 149 ; P. Grimal, La littérature latine, Paris, PUF, 1965 [1992], p. 88. On cite à l'appui de cette hypothèse Quintilien $(10,1,39)$, Sénèque le Rhéteur (Controverses, X, 2 , 26 ; IX, 24, 14), Tacite (Annales, 4, 34, 3).

23. M. Mahé-Simon, «L'enjeu historiographique de l'excursus sur Alexandre (IX, 16, 11-19, 17)», dans D. Briquel, J.-P. Thuillier, Le Censeur et les Samnites. Sur Tite-Live, livre IX, Paris, Éditions Rue d'Ulm, 2001, p. 37-63 ; A. Rossi, «Parallel Lives : Hannibal and Scipio in Livy's Third Decade», Transactions of the American Philological Association, 134, 2004, p. 358-381.

24. Texte de W. R. Paton (Polybius. The Histories. Vol. V. With an English Translation by W. R. P., Cambridge (Mass.)-Londres, Loeb Classical Library, 1926 [2006]) ; les traductions sont pour la plupart empruntées à D. Roussel (Polybe. Histoire, Paris, Gallimard, 2003) et ont parfois été légèrement modifiées.

25. Sur les propositions de restitutions de ce passage, voir F. W. Walbank, A Historical Commentary on Polybius. Vol. II, Oxford, Clarendon Press, 1967, p. 573.

26. Sur la compréhension de ce paragraphe, voir F. W. Walbank, op. cit., p. 573-574.

27. Il s'agit là du principe fondateur des différentes formes d'éloges (L. Pernot, La rhétorique de l'éloge dans le monde gréco-romain, Paris, Institut d'études augustiniennes, 1993, I, p. 133), qu'on retrouve également énoncé par Théon pour le parallèle (Progymnasmata, 10, 80 Patillon).

28. Voir sur ce point, le corrigé proposé par Aphthonios (Progymnasmata, 10, 4-8, 141-143 Patillon) ou les exemples attribués à Libanios (C. A. Gibson, Libanius's Progymnasmata: Model Exercises in Greek Prose Composition and Rhetoric. Translated with an Introduction and Notes by C. A. G., Atlanta, Society of Biblical Literature, 2008).

29. Texte et traductions tirés de G. Achard, Tite-Live. Histoire romaine. T. 23. Livre XXXIII. Texte traduit et établi par G. A., Paris, CUF, 2002.

30. Le texte est ici reconstruit à partir de celui de Polybe ( $c f$. J. Briscoe, A Commentary on Livy. Books XXXI-XXXIII, Oxford, Oxford University Press, 1973, p. 255).

31. J.-B. Grize, Logique et langage, Paris, Ophrys, 1990, p. 48.

32. Texte de M.Patillon, Corpus Rhetoricum. T.1. Préambule à la rhétorique. Aphthonios, Progymnasmata. Pseudo-Hermogène, Progymnasmata, Paris, CUF, 2008, p. 141 (traduction personnelle).

33. Voir note 43. 
34. P. Pédech, op. cit., p. 420. D'autres récits chez Polybe semblent également adopter le mode du parallèle, comme celui de la bataille de Drépane $(1,51)$, des îles Égates $(1,61)$ et de Zama $(15,9$ $16,6)$.

35. F. W. Walbank, op. cit., p. 579.

36. Tite-Live $(33,7,13)$ est plus succinct. Cf. J. Briscoe, op. cit., p. 257.

37. F. W. Walbank, op. cit., p. 580.

38. F. W. Walbank, op. cit., p. 584.

39. Pour une étude plus détaillée du personnage de Flamininus dans ce passage et les différences dans la représentation de la bataille, voir E. Carawan, "Graecia Liberata and the Role of Flamininus in Livy's Fourth Decade ", Transactions of the American Philological Association, 118, 1988, p. 209-252 ; T. J. Luce, Livy: The Composition of his History, Princeton, Princeton University Press, 1977, p. 39-41 ; H. Tränkle, op. cit., p. 100-102 et 187-189 ; B. Sans, « De Zama à Cynoscéphales », op. cit.

40. Cf. Tite-Live, 33, 6, 12. L'historien précise à deux reprises que Flamininus cherche à se prémunir des embuscades de l'ennemi, ce qui contribue à dépeindre un chef conscient du danger et un ennemi sournois.

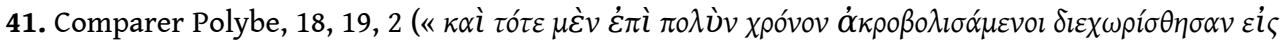
$\tau \grave{\alpha} \varsigma \alpha \dot{v} \tau \tilde{\omega} v \pi \alpha \rho \varepsilon \mu \beta 0 \lambda \alpha ́ \varsigma$ ", " après des escarmouches prolongées, les deux troupes se séparèrent pour l'heure et regagnèrent leurs camps respectifs ») et Tite-Live, 33, 6, 6 («fugati atque in castra compulsi sunt ", « les soldats du roi furent mis en fuite et repoussés dans leur camp ») ou encore

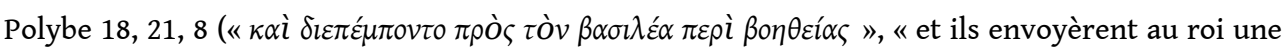
demande d'aide ») et 33, 7, 8 (« opem regis per nuntios implorabant», « ils imploraient l'aide du roi par l'intermédiaire de messagers »). Cf. G. Achard, op. cit., p. 76 ; J. Briscoe, op. cit., p. 257.

42. Cf. Tite-Live, 33, 8, 2 : « inuitum et cunctabundum et dicentem <Philippum > temere fieri, non locum sibi placere non tempus », " malgré lui Philippe qui hésitait, qui disait que l'on agissait à la légère, que ni le lieu, ni le moment ne lui convenaient »); le verbe trepidare («trembler ») apparaît deux fois $(33,7,8 ; 8,10)$ pour qualifier son comportement.

43. Elle ne profite pas en effet du principal atout tactique signalé par Polybe, puisque dans le texte latin $(33,8,13)$, elle a dû renoncer à ses lances, trop encombrantes pour combattre ("quarum longitudo impedimento erat», "dont la longueur était gênante »). On considère généralement que cette information, qui n'apparaît que chez l'historien latin, résulte d'une erreur de traduction (G. Achard, op.cit., p. 78; H. Tränkle, op. cit., p.180; T.J. Luce, op. cit., p. 39-40, p.171 ; J.Briscoe, op.cit., p. 263 ; F.W.Walbank, op.cit., p. 582 ; P.G. Walsh, "The Negligent Historian : 'Howlers' in Livy ", Greece \& Rome, 5, 1958, p. 84-88).

44. Tite-Live, 18, 9, 3-4: «À l'aile droite (dextero cornu), le roi, aidé surtout par le terrain, puisqu'il luttait en descendant de la montagne, l'emportait ; à l'aile gauche (sinistro), c'était la panique et le désordre car la partie de la phalange qui avait été mise à l'arrière était justement en train d'arriver ; le centre (media acies), qui était le plus près de l'aile droite, restait immobile comme s'il regardait un combat qui ne le concernait pas. La phalange qui était arrivée en colonne plutôt qu'en ligne de bataille, et qui était dans une formation plus adaptée à la marche qu'au combat, était à peine parvenue au sommet. » Les expressions dextero cornu, sinistro et media acies semblent indiquer que Tite-Live applique mécaniquement le schéma stéréotypé d'une bataille rangée classique, ce qui ne correspond sans doute pas ici à la réalité (G. Achard, op. cit., p. 78 ; T. J. Luce, op.cit. p.39-40 ; J. Briscoe, op.cit., p. 264 ; P.G.Walsh, Livy. His Historical Aims and Methods, Cambridge, Cambridge University Press, 1961, p.161, p.198-199), mais outre son intérêt didactique, cette division permet à Tite-Live de contrebalancer le succès de Philippe (comparer avec Polybe 18, 25, 2-3, où la situation semble plus indécise).

45. C'est en fait la conclusion à laquelle arrive Pédech (op. cit., p. 407, p. 431) sur la fonction des nombreux parallèles chez Polybe. Mais, d'une part, rien ne permet d'exclure que cette faculté 
n'est pas exercée par la rhétorique, bien au contraire. D’autre part, la version livienne témoigne d'un autre usage du parallèle.

46. Pour rappel, Polybe $(18,28,6-11)$ exclut cette hypothèse d'explication.

47. Ceci montre le caractère personnel, voire obsessionnel, de certaines vues ou de certains schèmes de pensées de l'historien grec. Cf. E. Nicholson, "Assessing and Assembling True Historiography: Polybius on Probability and Patterns", dans Th. Blank, F. Maier, Die symphonischen Schwestern. Narrative Konstruktion von ,Wahrheiten in der nachklassischen Geschichtsschreibung, Stuttgart, Franz Steiner, 2018, p. 187-207.

48. M.-R. Guelfucci, «Polybe, le regard, la structure des Histoires et la construction du sens », Cahiers des études anciennes, 47, 2010, p. 329-357.

49. Sur cette technique, enseignée dans le cadre des progymnasmata, voir R. Webb, Ekphrasis, Imagination and Persuasion in Ancient Rhetorical Theory and Practice, Farnham, Ashgate, 2009.

\section{AUTEUR}

\section{BENOÎT SANS}

Université libre de Bruxelles 